\title{
A Review of Uncertainties in Global Temperature Projections over the Twenty-First Century
}

\author{
R. Knutti ${ }^{\mathrm{a}, \mathrm{b}}$ M. R. Allen, ${ }^{\mathrm{c}}{ }^{\text {P. Friedlingstein, }}{ }^{\mathrm{d}}$ J. M. Gregory,,${ }^{\mathrm{e}, \mathrm{f}}$ G. C. Hegerl,${ }^{\mathrm{g}}$ G. A. Meehl, ${ }^{\mathrm{b}}$ \\ M. Meinshausen, ${ }^{\mathrm{h}}$ J. M. Murphy,${ }^{\mathrm{f}}$ G.-K. Plattner,${ }^{\mathrm{i}, \mathrm{j}}$ S. C. B. Raper,${ }^{\mathrm{k}}$ T. F. Stocker,${ }^{\mathrm{i}}$ P. A. Stott ${ }^{\mathrm{f}}$ \\ H. TENG, ${ }^{\mathrm{b}}$ AND T. M. L. WigLeY ${ }^{\mathrm{b}}$ \\ a Institute for Atmospheric and Climate Science, Zurich, Switzerland \\ ${ }^{\mathrm{b}}$ National Center for Atmospheric Research, ${ }^{1}$ Boulder, Colorado \\ ${ }^{\mathrm{c}}$ Department of Physics, University of Oxford, Oxford, United Kingdom \\ ${ }^{\mathrm{d}} I P S L / L S C E$, Gif-sur-Yvette, France \\ ${ }^{\mathrm{e}}$ Walker Institute, University of Reading, Reading, United Kingdom \\ ${ }^{\mathrm{f}}$ Met Office Hadley Centre, Exeter, United Kingdom \\ ${ }^{\mathrm{g}}$ School of Geosciences, University of Edinburgh, Edinburgh, Scotland \\ ${ }^{\mathrm{h}}$ Potsdam Institute for Climate Impact Research, Potsdam, Germany \\ ${ }^{\mathrm{i}}$ Climate and Environmental Physics, University of Bern, Bern, Switzerland \\ ${ }^{\mathrm{j}}$ Environmental Physics, Institute for Biogeochemistry and Pollutant Dynamics, ETH Zurich, Zurich, Switzerland \\ ${ }^{\mathrm{k}}$ CATE, Manchester Metropolitan University, Manchester, United Kingdom
}

(Manuscript received 27 June 2007, in final form 24 October 2007)

\begin{abstract}
Quantification of the uncertainties in future climate projections is crucial for the implementation of climate policies. Here a review of projections of global temperature change over the twenty-first century is provided for the six illustrative emission scenarios from the Special Report on Emissions Scenarios (SRES) that assume no policy intervention, based on the latest generation of coupled general circulation models, climate models of intermediate complexity, and simple models, and uncertainty ranges and probabilistic projections from various published methods and models are assessed. Despite substantial improvements in climate models, projections for given scenarios on average have not changed much in recent years. Recent progress has, however, increased the confidence in uncertainty estimates and now allows a better separation of the uncertainties introduced by scenarios, physical feedbacks, carbon cycle, and structural uncertainty. Projection uncertainties are now constrained by observations and therefore consistent with past observed trends and patterns. Future trends in global temperature resulting from anthropogenic forcing over the next few decades are found to be comparably well constrained. Uncertainties for projections on the century time scale, when accounting for structural and feedback uncertainties, are larger than captured in single models or methods. This is due to differences in the models, the sources of uncertainty taken into account, the type of observational constraints used, and the statistical assumptions made. It is shown that as an approximation, the relative uncertainty range for projected warming in 2100 is the same for all scenarios. Inclusion of uncertainties in carbon cycle-climate feedbacks extends the upper bound of the uncertainty range by more than the lower bound.
\end{abstract}

\section{Introduction}

Climate models forced with changing anthropogenic greenhouse gas, sulfur dioxide, and other aerosol emis-

\footnotetext{
${ }^{1}$ The National Center for Atmospheric Research is sponsored by the National Science Foundation.

Corresponding author address: R. Knutti, Institute for Atmospheric and Climate Science, Universitätstrasse 16, ETH Zurich, CH-8092, Switzerland.

E-mail: reto.knutti@env.ethz.ch
}

DOI: 10.1175/2007JCLI2119.1

(C) 2008 American Meteorological Society sions project global-mean surface air temperature to increase substantially in the future. The range of uncertainty given in the Intergovernmental Panel on Climate Change (IPCC) Third Assessment Report (TAR) for the end of the twenty-first century (2100 relative to 1990) was $1.4^{\circ}-5.8^{\circ} \mathrm{C}$ (Cubasch et al. 2001) for the Special Report on Emissions Scenarios (SRES) nonintervention emissions scenarios (Nakicenovic and Swart 2000). However, the interpretation of that temperature range is difficult for at least two reasons. First, it is a range produced by several models, with no indication of a likelihood distribution, and no confidence attached 
to the range (e.g., the probability for a temperature rise above that range is unknown). Second, the range combines uncertainties in both emission scenarios and climate model responses and gives no clear indication of the relative importance of these two quite distinct types of uncertainty. Uncertainties in the response of the climate system to a given emissions scenario, dominated by uncertainties in the climate sensitivity and the rate of ocean heat uptake, are determined by our incomplete understanding of physical processes and the limitations of climate models. The strength of the relevant climate feedbacks cannot significantly be influenced by human activities. The uncertainties in emissions, on the other hand, are an uncertainty related to human actions and decisions, and in that sense are a choice rather than an intrinsic uncertainty. It is therefore important to consider the uncertainty in the climate response separately for each emissions scenario (Knutti et al. 2002; Wigley 2004).

While in a subjectivist Bayesian framework, likelihoods or conditional probabilistic distributions of warming for a given scenario may be estimated by standard methods, deriving likelihoods of warming across scenarios is more problematic, because no likelihoods have been attached to any of the individual SRES scenarios. In the absence of such information, equal probability has sometimes been assumed for each scenario (see e.g., Wigley and Raper 2001). Another limitation of analyses based on the SRES scenarios is that they assume no efforts to mitigate climate change, and thus at least on the lower bound do not span the full economically and technically feasible range of future emission pathways that mitigation would allow.

Progress has been made since the publication of the IPCC TAR (Houghton et al. 2001) in quantifying and understanding uncertainties in future temperature projections. Probabilistic projections with a variety of models of different complexity and different statistical methods have emerged, using large ensembles of simplified models, statistical emulators, and combining observations with models using Bayesian methods. Climate responses have been simulated with a larger number and more comprehensive atmosphere-ocean general circulation models (AOGCMs) than ever before, and uncertainties in carbon cycle-climate feedbacks (i.e., the fact that ocean and terrestrial carbon uptake depends on the climatic state) have been estimated using models of different complexities.

Here we present global temperature projections from the most recent coordinated model intercomparison performed for the IPCC Fourth Assessment Report (AR4; Meehl et al. 2007), and identify the robust conclusions as well as the differences arising from the use of different probabilistic methods. We provide a more comprehensive discussion than in AR4 in order to achieve a more in-depth view on the various methods; where and why they agree or differ; expand on how the newer AOGCM projections compare to earlier ones; show why the AR4 results differ from the TAR; and quantify the contributions of scenario, climate feedback, carbon cycle feedback, and structural uncertainty to the total uncertainty. We show that relative uncertainties are the same for different SRES emissions scenarios, independent of the total amount of greenhouse gas emissions.

\section{Results}

\section{a. Probability density functions and ranges from individual methods}

In the large coordinated modeling effort of the World Climate Research Coupled Model Intercomparison Project phase 3 (WCRP CMIP3), in preparation for the IPCC AR4, 23 AOGCMs have simulated climate change over the twentieth century and made projections into the future for low, medium, and high nonintervention emissions scenarios (SRES B1, A1B, and A2, respectively) that are based on different assumptions about population growth, economic development, energy use, and globalization. Although problematic for reasons detailed below, the multimodel mean and standard deviation of global temperature change are shown in Fig. 1. The ranges in that figure are best seen as an illustration of uncertainty ranges, despite the fact that the figure uses the most comprehensive models available. The main difficulties in interpreting an ensemble of opportunity are the following. First, the set of models represents an ensemble of opportunity, neither sampled randomly nor systematically, and is not designed to span an uncertainty range. Different models have different climate sensitivities and the range and distribution of these sensitivities differ from ranges and distributions estimated from observational data. Second, the models are not all independent (Tebaldi and Knutti 2007). Third, not all models have simulated all scenarios. This results in the spread for the higher scenario A2 apparently being smaller than for the lower scenario B1. However, for the subset of models that have run both scenarios, the spread is larger for A2 than for B1, consistent with the constant relative uncertainty argument further discussed below. Fourth, different models use different sets of forcings, and they quantify the common forcings differently (Collins et al. 2006; Forster and Taylor 2006). Fifth, it seems important to note that for prescribed emissions, carbon cycle processes, themselves dependent on the climate change 

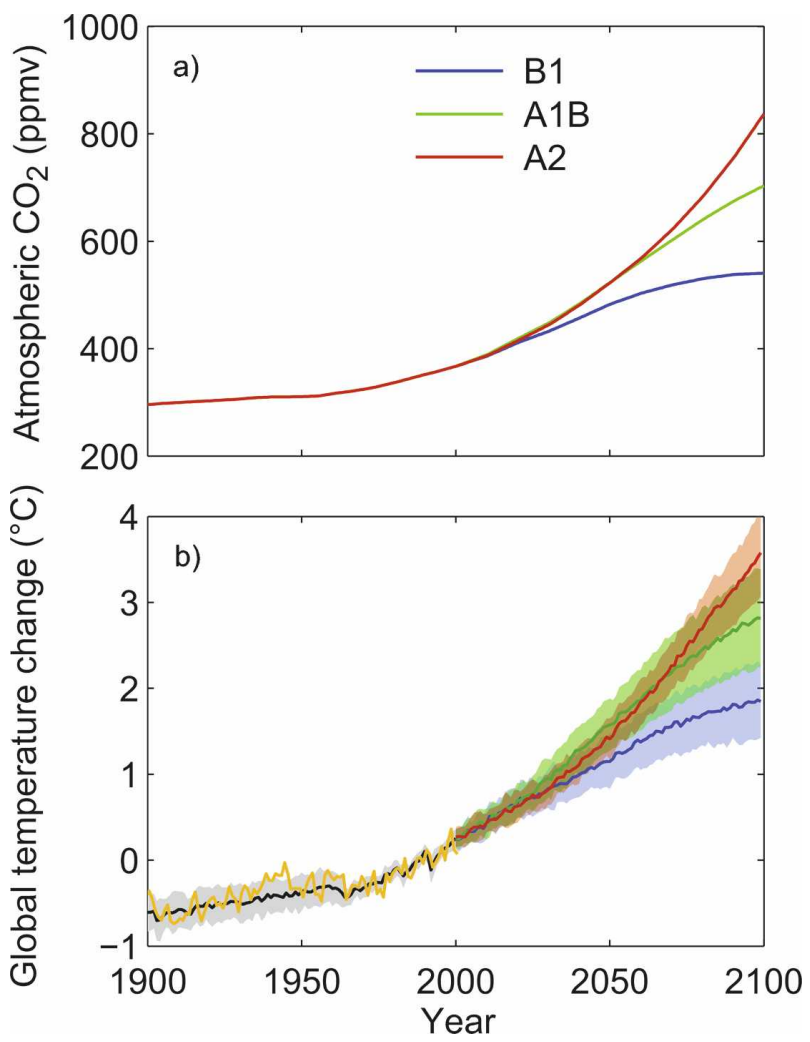

FIG. 1. (a) Atmospheric carbon dioxide concentration for the SRES scenarios B1 (blue), A1B (green), and A2 (red) derived with the BernCC model (Joos et al. 2001) for the TAR [Houghton et al. 2001; and used to force most of the simulations in (b)]. Note that the scenarios prescribe emissions, and atmospheric concentrations and radiative forcings considered in the AOGCMs vary. (b) Global-mean surface air temperature anomalies from the 1980-99 average for SRES scenarios B1 (blue), A1B (green), A2 (red), and for the historic twentieth-century simulation (black). Lines denote the ensemble mean and shaded bands denote one standard deviation of the multimodel response. Ensemble members were averaged first for each model, such that each model is given equal weight, although note that not all simulations are available for all models. The observed temperature for the twentieth century is given in orange for comparison (Jones and Moberg 2003).

response, substantially affect the modeled atmospheric $\mathrm{CO}_{2}$ concentration (Friedlingstein et al. 2006) and thus radiative forcing, yet all AOGCMs considered here prescribe atmospheric $\mathrm{CO}_{2}$ from offline carbon cycle simulations, and many of them use the same atmospheric concentration scenario. While they all account for carbon cycle-climate feedback uncertainties, their projected temperature range is smaller than it would be if they all had accounted for the uncertainty in the carbon cycle-climate feedbacks.

Physical feedbacks determine the temperature response to a given atmospheric concentration. This temperature response can be characterized by the equilibrium climate sensitivity (the global equilibrium surface temperature response to a doubling of atmospheric $\mathrm{CO}_{2}$ ) and the transient climate response (TCR; the global surface temperature response at the time of $\mathrm{CO}_{2}$ doubling in a scenario where atmospheric $\mathrm{CO}_{2}$ increases by $1 \% \mathrm{yr}^{-1}$, i.e., in a 20 -yr mean period centered at year 70). By definition, equilibrium climate sensitivity and TCR do not include carbon cycle feedbacks, because both concepts connect temperature changes to a given $\mathrm{CO}_{2}$ concentration. The effect of the changing climate on the carbon cycle is treated as a feedback and is considered in all climate change projections presented here. While a temperature response to a given emission can be defined (i.e., combining the physical and biogeochemical feedbacks) as a reference number (like climate sensitivity describing only the physical feedbacks), it is of limited use, since the temperature response to a given emission depends both on the time and the climate state.

The difficulty in interpreting the TAR results in terms of probability has prompted several attempts to provide probability density functions (PDFs) of global temperature increase. Note that each of these distributions is of course conditional on the model, method, and data constraint that are used, and that the percentiles of the distributions reflect a degree of belief that warming would fall within certain temperature ranges if we were to follow these scenarios, rather than a frequency of occurrence in an experiment that is repeated many times. Figure 2 (left column, lower part of each panel) summarizes the available cumulative distribution functions (CDFs), for the six illustrative SRES scenarios, for the early (2020-29, dashed CDFs) and latetwenty-first century (2090-99, solid CDFs), relative to the 1980-99 average. In short, Wigley and Raper (2001) produce PDFs from a large ensemble with a simple upwelling diffusion energy balance model, varying climate sensitivity, ocean vertical diffusivity, carbon cycle feedbacks, and aerosol forcing. Parameters are varied in ranges considered plausible by the authors (most ranges were taken from the IPCC TAR); no formal observational constraints are applied. The uncertainty in climate sensitivity (being the largest contribution to the total uncertainty for a given scenario) is represented as a lognormal distribution where the $1.5^{\circ}-4.5^{\circ} \mathrm{C}$ range corresponds to the $90 \%$ confidence interval. Knutti et al. (2003) consider uncertainties in climate sensitivity, ocean diffusivity, and different mixing parameterizations (e.g., horizontal-vertical diffusion, isopycnal diffusion, and Gent-McWilliams mixing; Knutti et al. 2000), carbon cycle feedbacks, and all radiative forcing components individually in an intermediate complexity climate model (EMIC) with a dynamical ocean and an energy balance atmosphere, and con- 

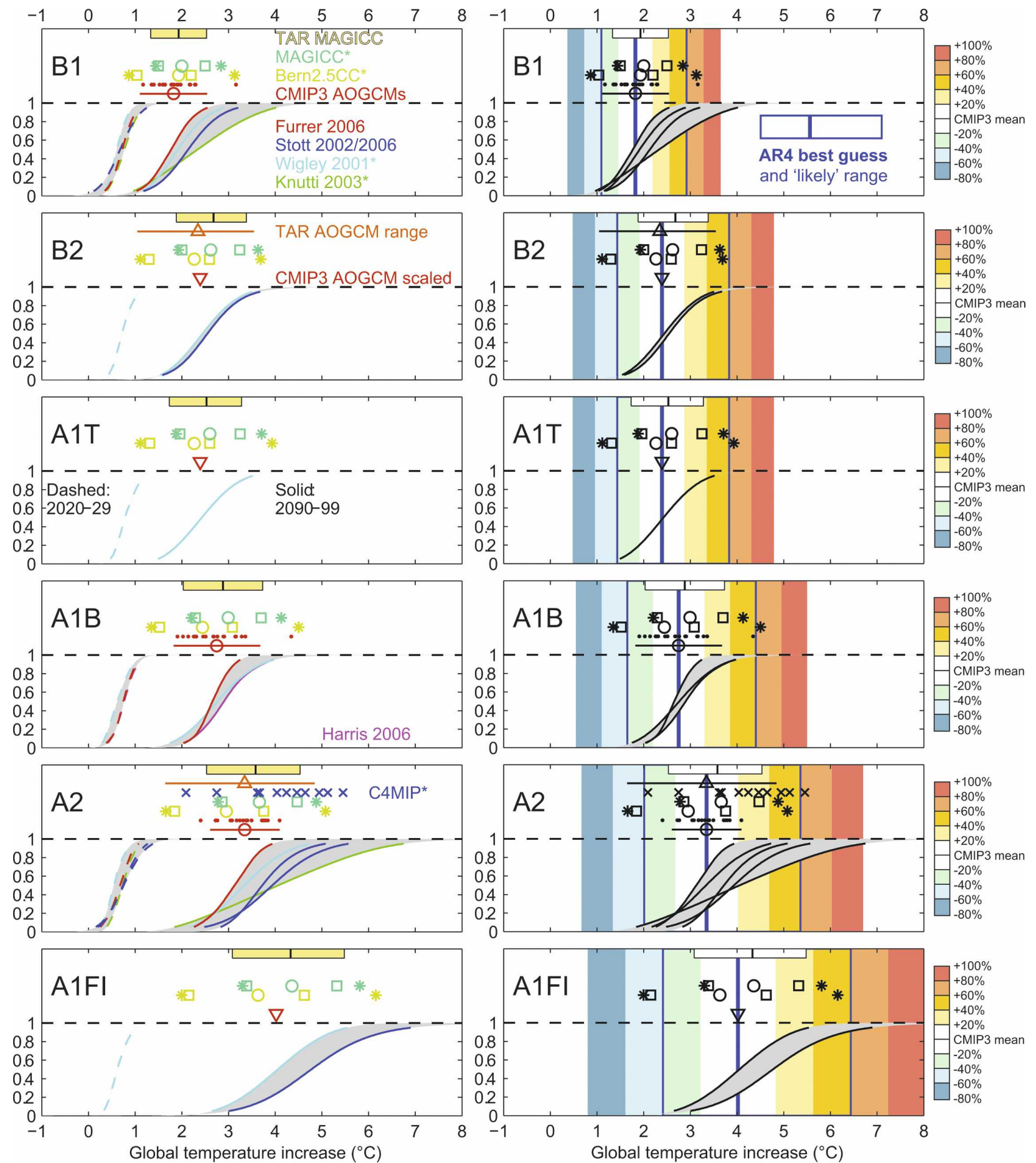

FIG. 2. Global-mean temperature projections for the six illustrative SRES scenarios. (left) The CDFs for global-mean surface air temperature increase for short-term (2020-29, dashed) and long-term (2090-99, solid) projections relative to the 1980-99 average are shown at bottom of each plot. Different colors mark different models or statistical methods. Lines are only shown from the $5 \%$ to the $95 \%$ level, thus the horizontal dimension of each curve marks the 5\%-95\% confidence range. Light gray shading marks the envelope of all CDFs. Labels with asterisks indicate that the respective method considers carbon cycle-climate feedback uncertainties. Other estimates of uncertainty for 2090-99 are shown at the top of each plot. Central values and ranges are given as means and 5\%-95\% from a fitted normal distribution to the AR4 AOGCM simulations (red circles and lines) for B1, A1B, and A2, along with results for each individual AR4 AOGCM (red dots). For B2, A1T, and A1FI, AOGCM means are estimated using scaling factors from the MAGICC model (red triangles, see text). From the MAGICC simple model (dark green symbols) tuned to 19 AR4 AOGCMs, means (circles) and one standard deviations (squares) are given for fixed carbon cycle parameters (indicating the uncertainty caused by the climate 
strain the multithousand-member ensemble with the observed global surface warming (Jones and Moberg 2003) and ocean heat uptake (Levitus et al. 2000). A uniform prior distribution from $1^{\circ}$ to $10^{\circ} \mathrm{C}$ is assumed for climate sensitivity. Because climate sensitivity is poorly constrained from the observed global-mean warming trends (Forest et al. 2002; Knutti et al. 2002; Frame et al. 2005) and the use of a uniform prior distribution on climate sensitivity, that study finds larger probabilities for high warming than most others. The simplified models used in these studies have the advantage that a large portion of the parameter space can be explored, parameters like climate sensitivity can be adjusted to match specific distributions, and very large ensembles of simulations can be generated. On the other hand, those types of models inevitably have to simplify the description of feedback processes.

Stott and Kettleborough (2002) and Stott et al. (2006) use a detection-attribution method to derive best-guess scaling factors and uncertainty ranges, by which model-simulated greenhouse gas and aerosol responses each have to be multiplied to best match twentieth-century climate change. These scaling factors are then used to estimate the best estimate and uncertainty range of future change and account for uncertainties in the estimate of greenhouse gas forcing to twentiethcentury change caused by internal variability and uncertainty in natural forced variability as well as for uncertainty in climate sensitivity and ocean heat uptake. Carbon cycle uncertainties are not considered. This method does not explicitly prescribe a prior distribution for climate sensitivity, but in effect it implicitly uses a uniform prior on TCR [or equivalent, the greenhouse gas attributable warming, see also Frame et al. (2006)], which a priori assigns a lower probability to high values of climate sensitivity than what the uniform prior on climate sensitivity (Knutti et al. 2002) does. Therefore, for long-term projections where climate sensitivity be- comes increasingly important, the Stott et al. (2006) method tends to assign lower probability to very high warming. The use of more detailed information and a noise reducing metric on surface temperature changes also leads to reduced uncertainty and a narrower PDF. The detection method is based on one or more AOGCMs, which is a more accurate representation of the climate system than the simple models or EMICs, but it has to assume a certain linearity in the way model mismatch against observations over the past decades projects into the future.

Furrer et al. $(2007 a, b)$ use a Bayesian spatial hierarchical method to obtain PDFs at each grid point from the set of AR4 AOGCMs. The assumptions are that the response from each AOGCM can be decomposed into the "true" underlying large-scale climate change signal plus a small-scale noise component representing model error and internal variability. The noise component is specific to each model, but is assumed to have zero average across all models. The climate change signal is decomposed into a series of spherical harmonics and corresponding coefficients, along with their uncertainties. By recombining the PDFs of the coefficients with the basis functions, a joint PDF of the true climate change signal at each location can be obtained. The results from that method are based on the most complete and recent set of models, which in contrast to all other methods samples structural uncertainty in the models to some degree. However, the results share the limitations in the underlying multimodel ensemble dataset noted above; namely, that the range of AOGCMs may not adequately sample the range of uncertainty that we think exists. The implicit underlying distribution of climate sensitivity is the one of the CMIP3 AOGCM ensemble.

Harris et al. (2006) combine a 17-member perturbed physics ensemble of the fully coupled Hadley Centre coupled climate model version 3 (HadCM3) AOGCM

\footnotetext{
$\leftarrow$

only), and one standard deviation including carbon cycle uncertainties (asterisks). For the Bern2.5CC EMIC (light green symbols), the central values use a medium climate sensitivity and standard carbon cycle settings (circles), uncertainties are estimated by combining a high and low climate sensitivity with standard carbon cycle settings (squares) and by combinations of a low climate sensitivity and an efficient carbon cycle (i.e., removing excess carbon quickly from the atmosphere), and a high climate sensitivity and an inefficient carbon cycle (asterisks) to sample the full range. Individual model results are shown for the C4MIP models for A2, accounting for non- $\mathrm{CO}_{2}$ forcings (blue crosses). Model means and minimum maximum ranges (yellow bars) given in the TAR (Houghton et al. 2001) resulting from the tuning of the MAGICC model to seven TAR AOGCMs, as well as means and minimum to maximum ranges from nine AOGCMs shown in the TAR (Houghton et al. 2001; orange triangles and lines), are given for comparison. Ranges have been adjusted to match the periods 2090-99 and 1980-99, and may slightly differ from the original publications. Note that some ranges are simply minimum to maximum ranges from a set of models, or sensitivity tests of a model, and thus cannot be interpreted as percentiles of a distribution. (right) The color bands show ranges of constant relative uncertainty around the CMIP3 AOGCM mean for all scenarios, along with the individual estimates from the (left). A likely range of $-40 \%$ to $+60 \%$ (blue box) around the CMIP3 AOGCM mean (thick blue line) was given in IPCC AR4 (Meehl et al. 2007) for the purpose of giving policy-relevant advice, based on expert judgment and all the individual uncertainty estimates reviewed here.
} 
with an energy balance model and a larger ensemble of slab simulations of the Hadley Centre coupled atmospheric model version 3 (HadAM3) to emulate the probabilistic response of a large perturbed physics ensemble. As the Furrer et al. (2007b) method, this Hadley Centre ensemble (Harris et al. 2006) does not consider carbon cycle uncertainties.

Both the new AOGCM results and the probabilistic studies confirm that the anthropogenically forced shortterm temperature response (i.e., ignoring the possibility of several large volcanic eruptions in the near future, dashed CDFs) is quite well constrained (in terms of absolute uncertainty), with relatively consistent results across different methods. Temperature changes are almost independent of the scenario for the first few decades of the projections. This is because the scenarios are similar in terms of emissions at the beginning, and because part of the short-term warming is a delayed response to radiative forcing caused by past emissions. About half of the warming over the next few decades would occur even if the levels of atmospheric constituents and thus radiative forcing were held constant at year 2000 values (constant composition commitment; Meehl et al. 2005; Wigley 2005; Meehl et al. 2007). This commitment idea (i.e., that warming will continue even if the forcing is held constant) has been recognized long ago (Hansen et al. 1984; Siegenthaler and Oeschger 1984; Wigley and Schlesinger 1985; Schlesinger 1986). Also, some climate feedbacks or nonlinear interactions only become important on longer time scales.

The similarity of the short-term projections must not be misinterpreted as an argument that the choice of the scenario is unimportant for climate change. On the contrary, it highlights the long time scales of the climate system, implying that scenario choices today have an effect in the far future (e.g., Hansen et al. 1988; Wigley et al. 1996; Knutti et al. 2002; Stott and Kettleborough 2002). The increasing importance of scenario dependence over time arises because the temperature response of the system is determined by the stock of atmospheric greenhouse gases, rather than the flows represented by annual emissions. A consequence of this is that mitigation efforts must be started soon to become effective in the long term and avoid large (and potentially very high impact) climate change, since much more drastic mitigation efforts would be needed later to avoid large future changes. Because of the long time scales involved in the carbon cycle and the deep ocean, it will take centuries or millennia, even for substantial emission reductions, for the climate system to return from high levels of atmospheric concentrations (e.g., $700 \mathrm{ppm}$ in SRES A1B year 2100) to a state similar to that of today (Plattner et al. 2008).
The distributions for global-mean temperature change shown in Fig. 2 are close to Gaussian and do not show the pronounced long tail seen in many PDFs of climate sensitivity (e.g., Andronova and Schlesinger 2001; Forest et al. 2002; Knutti et al. 2002; Hegerl et al. 2006). This reflects the nonlinear relationship between TCR and climate sensitivity where the dependence of the transient response on changes in climate sensitivity reduces as the sensitivity increases (Hansen et al. 1984; Knutti et al. 2005; Allen et al. 2006). We note that the results depend on the assumed prior distribution for climate sensitivity, the choice of which is currently debated (Frame et al. 2005; Annan and Hargreaves 2006). Because of the nonlinear relationship between TCR and climate sensitivity, however, the effect is smaller than when attempting to constrain climate sensitivity itself. A renormalization of all results to the same prior distribution of climate sensitivity is not straightforward, and would be purely of reference value alone since any given prior distribution is contestable. Rational minds may disagree substantially regarding the form of the appropriate prior for climate sensitivity, so any choice of renormalization prior would be arbitrary. The results reviewed here are consistent with previous studies showing that transient climate change over the next century and the maximum warming under strong mitigation scenarios is better constrained by observations than the equilibrium warming in response to greenhouse gas (GHG) levels stabilized well above preindustrial levels (Knutti et al. 2005; Frame et al. 2006).

Despite the similarities in the shapes of the CDFs produced in different ways, there are noticeable offsets by up to $1^{\circ} \mathrm{C}$ for the high scenarios. The disagreement between different methods is consistently larger at the upper than the lower bound [i.e., the horizontal distance between different CDFs (indicated by the light gray shading) is larger at the $95 \%$ level than at the $5 \%$ level]. This highlights the importance of considering structural uncertainties (i.e., differences in the observational constraints used, the sources of uncertainties considered, choices in the statistical methodology, and single model biases persistent across whole ensembles) independent of the parameters used. Note that in all of those studies, simplifying the problem by neglecting certain sources of uncertainty (e.g., ignoring certain forcings, neglecting uncertainties in radiative forcing for given atmospheric concentrations; neglecting uncertainties in ocean mixing, climate feedbacks, or in carbon cycle parameters; not accounting for internal unforced variability) will almost always artificially reduce uncertainty ranges and lead to overly confident conclusions.

In addition to probabilistic results, Fig. 2 also shows the mean projections and one standard deviation 
ranges using the Model for the Assessment of Greenhouse-Gas Induced Climate Change (MAGICC; Wigley and Raper 2001), with climate feedback and ocean heat uptake tuned to the $1 \% \mathrm{yr}^{-1} \mathrm{CO}_{2}$ increase simulations of 19 CMIP3 AOGCMs, for a standard fixed carbon cycle and for carbon cycle feedbacks approximately covering the Coupled Carbon Cycle Climate Model Intercomparison Project (C4MIP; Friedlingstein et al. 2006) range. Similarly, projections with the University of Bern two-dimensional Carbon Cycle Climate Model (BERN2.5CC) EMIC model coupled to a simplified carbon cycle model (Joos et al. 1999, 2001; Plattner et al. 2001) are shown for climate sensitivities of $1.5^{\circ}$, $3.2^{\circ}$, and $4.5^{\circ} \mathrm{C}$ (approximately covering the likely climate sensitivity range given in the TAR), with standard carbon cycle settings and with carbon cycle parameters tuned for efficient and inefficient carbon removal from the atmosphere to the ocean and terrestrial biosphere.

The CMIP3 AOGCM results are also shown for the B1, A1B, and A2 scenarios. For B2, A1T, and A1FI, no AOGCM simulations are available, so the mean AOGCM response for the year 2100 is approximated by multiplying the mean AOGCM A1B response with the ratios of temperature change between scenarios $\mathrm{B} 2 /$ $\mathrm{A} 1 \mathrm{~B}, \mathrm{~A} 1 \mathrm{~T} / \mathrm{A} 1 \mathrm{~B}$, and $\mathrm{A} 1 \mathrm{FI} / \mathrm{A} 1 \mathrm{~B}$, respectively, derived from the MAGICC model. Note that the CMIP3 models prescribe greenhouse gas concentrations rather than emissions, and do not sample carbon cycle uncertainties. Results from C4MIP (Friedlingstein et al. 2006) are shown for the $\mathrm{A} 2$ scenario, accounting additionally for non- $\mathrm{CO}_{2}$ forcings (blue crosses). The minimum to maximum range of the TAR AOGCMs as well as the minimum to maximum range of the TAR MAGICC model results, derived from tuning to seven of the nine TAR AOGCMs, are shown for comparison. The raw TAR AOGCM range (Fig. 2, orange lines) is wider than the MAGICC range because MAGICC was not tuned to the highest and lowest AOGCM in the TAR.

Since the uncertainty grows with time and with increasing warming, it is instructive to study the relative uncertainty (i.e., the spread divided by the median versus time) for a few of the uncertainty estimates. Figure 3a shows that after about 2020, the relative uncertainty in the CMIP3 models (blue, for the subset of models that have run all three scenarios) is almost constant over time, and independent of the scenario. For the EMIC PDF (Knutti et al. 2003), relative uncertainty increases slightly with time, caused by very high climate sensitivities (red). If climate sensitivity is assumed to be below $4.5^{\circ} \mathrm{C}$ in that study (magenta), the relative uncertainty is almost constant over time. Note that this does not imply that this approach is a priori valid to derive uncertainties throughout the twenty-first century as will be detailed below. In the detection case (green; Stott et al. 2006), the uncertainty is larger in the early decades. Those results include a number of sources of uncertainty in future temperatures that are not included in some other uncertainty predictions. The predictions include uncertainty due to only having a small number of predictions from forecast ensembles of the forced response (often only one). In addition to the uncertainty in the underlying anthropogenically forced temperature change, they include uncertainty due to future natural forcing, estimated from model estimates of past variability in naturally forced temperatures. Finally, they additionally include the uncertainty due to internal variability on top of the forced response and also include the extra uncertainty from taking the difference between two imperfectly known decadal-mean temperatures. Further details are given in the methods section of Stott and Kettleborough (2002). The contributions from internal unforced and natural forced variability are constant over time, and thus their relative effect is larger in the early decades when the anthropogenic forced signal is still weaker. When considering only the forced response, the relative uncertainty in the early part of the century is about 0.4 , similar to the estimates from other methods.

Figure $3 \mathrm{~b}$ shows that for methods that do not assume Gaussian distributions, the ratio of the $5 \%$ and $50 \%$ percentile and the ratio of the $95 \%$ and $50 \%$ percentile are also remarkably constant and similar for the EMIC (magenta, for B1 and A2) and MAGICC PDFs (black, all six illustrative scenarios). The uncertainty relative to the median and variations across different scenarios are larger at the upper bound than at the lower.

While each method has its own characteristic shape for the evolution of uncertainty over time, they all agree that for a given method, the relative uncertainty toward the end of the century should be very similar for each scenario. This is because the ratio of temperature change to forcing is approximately constant across scenarios for the types of scenarios and time scales considered here. Note that the relative uncertainty for the higher emission scenario A2 is always slightly smaller than for $\mathrm{A} 1 \mathrm{~B}$ or $\mathrm{B} 1$, because for $\mathrm{A} 2$ the temperature signal is larger and because the relative uncertainty in the forcing is smaller due to the larger relative contribution of GHG forcing. When considering only the forced response, the relative uncertainty is also reasonably constant over time after 2020 .

Note that when estimating uncertainty in short-term projections (e.g., for the decade 2020-29) an additional difficulty arises that has not been considered so far in the literature. Projections are taken here as differences to the average over the $1980-99$ period, so effectively 

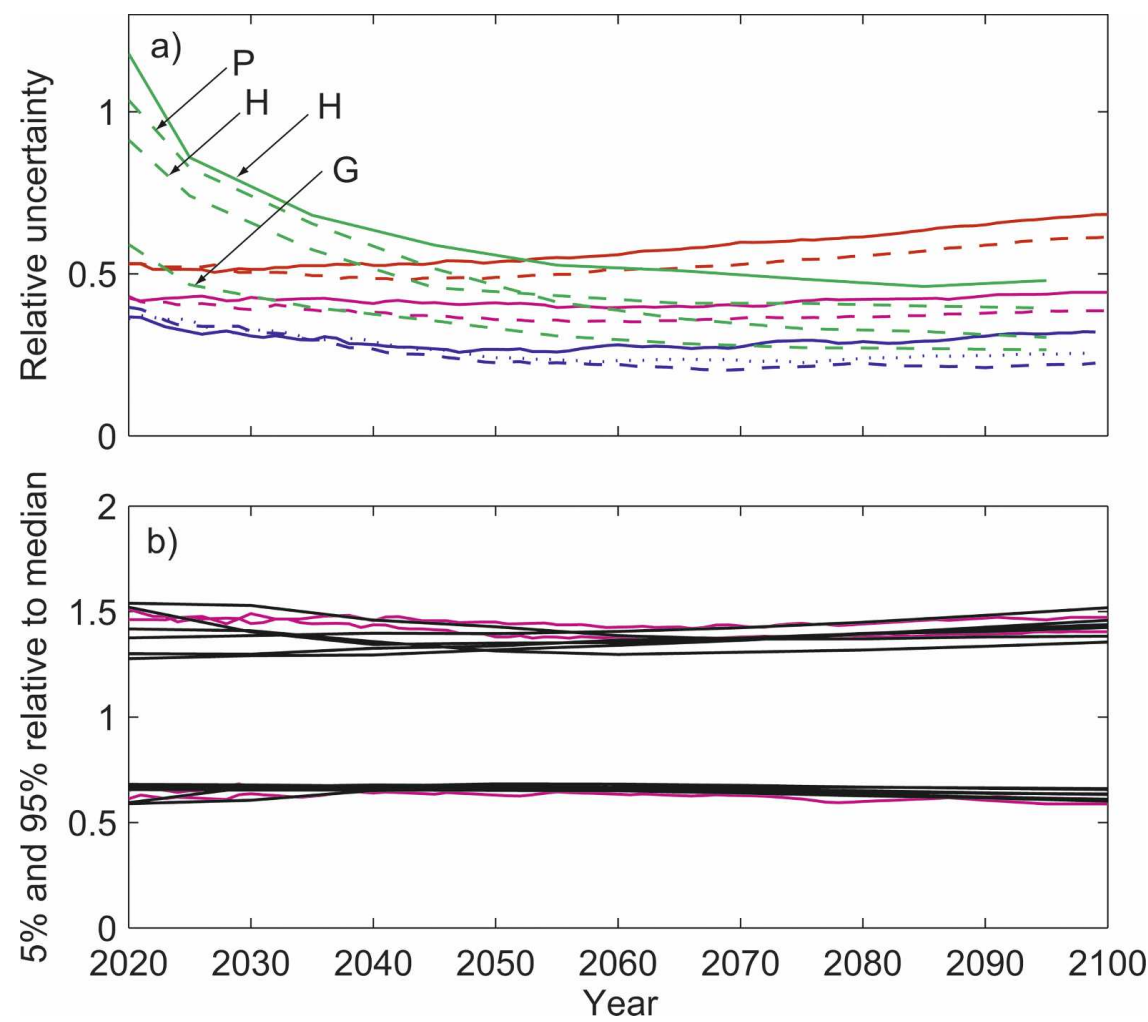

FIG. 3. (a) Relative uncertainty of global-mean surface air temperature increase above 1980-99 vs time for different models and methods. For PDFs, the relative uncertainty is defined here as half of the $5 \%-95 \%$ range, divided by the median of the PDF. For the AOGCMs, the relative uncertainty is taken as 1.65 times the standard deviation across the models divided by the mean. For a normal distribution, the two definitions are identical. The relative uncertainty is shown for scenarios B1 (solid), A1B (dotted), and A2 (dashed), for the subset of the AR4 AOGCMs (blue) that have run all three scenarios, for probabilistic projections with an EMIC (Knutti et al. 2003) for climate sensitivity constrained by observations (red) and climate sensitivity in the range of $1.5^{\circ}-4.5^{\circ} \mathrm{C}$ constrained by observations (magenta), and for the fingerprint scaling methods (Stott et al. 2006; green) using the HadCM3 (label H), NOAA/GFDL R30 (label G), and NCAR PCM (label P) AOGCMs. The Stott et al. results are shown as one value per decade; the other estimates are based on 10-yr running means to reduce noise. (b) The $5 \%$ and $95 \%$ percentiles each divided by the median for the EMIC PDF and scenarios B1 and A2 (magenta; Knutti et al. 2003) and for the MAGICC PDF for all six illustrative SRES scenarios (black; Wigley and Raper 2001).

this is like making a projection in year 1990 for 2025 (i.e., $35 \mathrm{yr}$ into the future). However, as of 2007, half of those $35 \mathrm{yr}$ have already passed, and the warming observed from the base period up to 2007 has not been considered in the projections. If the uncertainty is growing linearly as argued above, then the uncertainty estimate starting in the base period 1980-99 is likely to be too large, and it would be smaller if the observations until today had been used. While this is a small issue for projections for 2100 (Meehl et al. 2007), it should be kept in mind that for short-term projections, taking differences to some base period in the past is problematic. The problem gets worse the further back the base period is chosen. Near-term uncertainties will be overes- timated, even in the case where the trends of model and observations are very similar.

\section{b. Overall uncertainty range from multiple methods}

There is no formal statistical way to combine all the estimates summarized in Fig. 2, since they are not all independent, and do not all consider the same sources of uncertainty. One option is to resort to imprecise probability (e.g., Kriegler and Held 2005; Hall et al. 2007; Tomassini et al. 2007), that is, consider an uncertainty in PDFs or sets of PDFs. However, these secondorder uncertainties are not easy to understand for the nonexpert, and the dependencies of the different methods are an issue. 
The right column in Fig. 2 shows colored bands of constant relative uncertainty around the CMIP3 mean for each scenario, along with all the individual PDFs and ranges from the left column in Fig. 2 discussed above. On the basis of the evidence presented above in support of a time- and scenario-independent relative uncertainty, a range of $-40 \%$ to $+60 \%$ around the CMIP3 mean was adopted in the IPCC AR4 (Meehl et al. 2007) as a "likely" ( $>66 \%$ probability) range for the projected warming in 2100 , for the purpose of giving policy-relevant advice that is easy to understand. The $-40 \%$ to $+60 \%$ range adopted in AR4 (Meehl et al. 2007) is indicated by blue lines and represents one of several defensible syntheses of the uncertainty across different models and methods. While the model results support a scenario-independent relative uncertainty (Fig. 3), the choice of an overall range encompassing the results from different methods is partly subjective. While Fig. 2 can provide a summary and some guidance, it is left to the reader to make his/her own expert judgment about an overall uncertainty range.

When interpreting the results, it is important to note that not all results are necessarily equally credible, and there may be processes that are incorrect or missing in different climate models. In addition, the assumptions in each study need to be kept in mind. Knutti et al. (2003), for example, who show the highest upper bound at the end of the century, used a uniform prior in climate sensitivity and rather poor constraints, resulting in a large fraction of simulations with climate sensitivities up to $10^{\circ} \mathrm{C}$. A subsequent study with the same model has found stronger constraints on climate sensitivity (Tomassini et al. 2007), which would push the upper bound of the projections down. Projected ranges in Bayesian approaches also depend on the prior distributions (Frame et al. 2005; Tomassini et al. 2007), which are not identical in all studies, as discussed above. What seems undisputable from Fig. 2 is the fact that structural uncertainty (the differences across studies arising from different assumptions, constraints, and choices of models) is large. Any uncertainty estimate based on a single method or model, which does not explicitly account for that, is therefore likely to underestimate the uncertainty. Figure 2 also highlights that due to the lack of formal methods to aggregate results, the derivation of probabilistic projections based on multiple lines of evidence has more subjective elements to it than the calculation of a PDF from a single method.

\section{c. Differences between IPCC TAR and AR4 ranges}

The ranges adopted in AR4 (Meehl et al. 2007; Fig. 2, blue boxes in right column) based on the assumption of a constant relative uncertainty and using the combined information from many methods are substantially wider than the model ranges given in the TAR for individual scenarios (Fig. 2, yellow bars) for several reasons. Although the TAR projections did account for climate feedbacks on the carbon cycle, they did not consider uncertainties in these feedbacks. The TAR range was based on a simple model tuned to seven AOGCMs, but no attempt was made to apply observational constraints. In contrast, the AR4 uncertainty estimates are also based on observational constraints and thus consistent with past observed trends and patterns. While the observational constraints in some of the newer methods tend to decrease projection uncertainties, this is outweighed by considering carbon cycle and structural uncertainties, such that the overall uncertainty given here is larger than in the TAR. Structural uncertainties include specific assumptions in each probabilistic method (e.g., whether uncertainty in all forcing components or just in the aerosol forcing is considered), in the construction of the climate model (e.g., choice of cloud parameterization), and in the observational constraints that are used [e.g., whether the model results are compared to observed spatial patterns, or just global averages; see Tebaldi and Knutti (2007) for a review of open issues]. These uncertainties all cause differences in projections between multiple methods, such that an overall uncertainty estimate accounting for these structural uncertainties is generally wider than an estimate from a single method.

The central values given in AR4 for each scenario are lower than those in the TAR, but the differences are small, and they should not be interpreted as a revised understanding of the relevant processes. They are caused by the fact that the MAGICC model results (which are tuned to the idealized CMIP3 $1 \% \mathrm{yr}^{-1} \mathrm{CO}_{2}$ increase scenarios) are slightly higher than the CMIP3 results for the SRES projections mainly due to different forcing assumptions. The AOGCM mean was used in AR4 as the central value, while the MAGICC model results were used as the central numbers in the TAR.

Differences between MAGICC and the CMIP3 AOGCMs arise primarily from the fact that many AOGCMs do not prescribe all radiative forcing components, whereas MAGICC does. In particular, accounting for the indirect aerosol effect (which is not included in many AOGCMs) in MAGICC leads to a larger radiative forcing increase between the base period 1980-99 and the end of the century and causes the projected change for the years 2090-99 to be higher by a few tenths of a degree. Small differences can arise from the fact that the MAGICC climate feedback and ocean heat uptake is tuned to the $1 \% \mathrm{yr}^{-1} \mathrm{CO}_{2}$ increase rather than the scenario simulations. In addition, 
MAGICC emulates all available AOGCM models for each scenario, even if some models do not provide all scenarios. This issue of sampling is also present in the multimodel ensemble, which is a rather arbitrary snapshot in time of the models available for the assessment, and not necessarily representative of our understanding of all the uncertainties that exist. However, those effects are small, and the different assumptions in radiative forcing explain a large part of the differences between MAGICC and the AOGCMs.

Apart from differences between MAGICC and the AOGCMS, in theory, differences could arise from both forcings and feedbacks being different in the new CMIP3 AOGCM ensemble (see Fig. 1) and the AOGCM ensemble used in the TAR. The mean of the TCR (the global average surface warming at the time of $\mathrm{CO}_{2}$ doubling in an idealized scenario where $\mathrm{CO}_{2}$ increases by $1 \% \mathrm{yr}^{-1}$ ) is $1.8^{\circ} \mathrm{C}$, with a standard deviation of $0.4^{\circ} \mathrm{C}$. Both the mean and standard deviation of TCR are virtually identical in the old and new AOGCM ensemble. The TCR measures the strengths of the relevant feedback processes and transient ocean heat uptake, does not depend on the carbon cycle, and is almost unaffected by forcing uncertainties. Projections for the end of the twenty-first century for SRES scenarios are linearly related to the TCR. Therefore, despite considerable progress in model development and the availability of many more models for AR4 (Meehl et al.2007), the best estimate for the sum of all transient physical feedbacks remains unchanged.

The projections in the TAR did not consider volcanic and solar forcing during the twentieth century. The inclusion of these forcings and the fact that more models now consider indirect aerosol forcing leads to smaller net forcing over the twentieth century and a larger net forcing increase over the twenty-first century, and projected warming for the years 2090-99 relative to the base period should be slightly higher. But additional simple model simulations, however, show the effect to be small, about a tenth of a degree. Differences in how other forcings are accounted for, and their efficacies (Hansen et al. 2005) in the different models might lead to other small differences. Unfortunately, for some of the old and new AOGCMs the information provided by the modeling groups about what components of the radiative forcing were used, what their magnitude is, and how they were implemented, is incomplete and makes a detailed separation of all effects impossible.

In summary, the TAR AOGCM mean (for the scenarios where it is available) is very similar to the AR4 AOGCM mean for both the SRES projections and the transient climate response, suggesting that the sum of the physical feedbacks is similar, and the effects of dif-

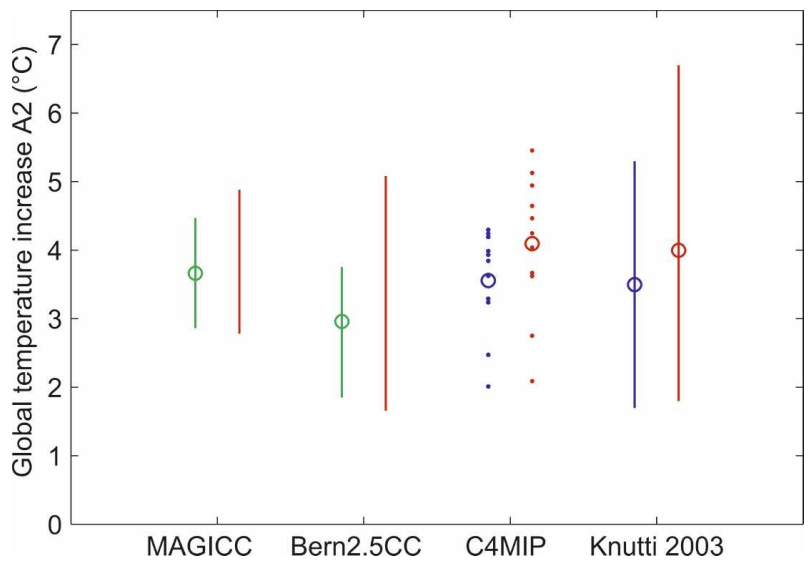

FIG. 4. Uncertainty in global-mean surface temperature increase for the end of the century (2090-99 relative to 1980-99) for the SRES A2 scenario. Blue, green, and red colors indicate no carbon cycle feedback (i.e., climate held constant for the carbon cycle), a nonzero carbon cycle feedback with constant parameters, and a nonzero carbon cycle feedback with uncertainty (perturbed parameters in the carbon cycle), respectively. Means and $1 \sigma$ ranges for the MAGICC and means and min-max ranges for the Bern2.5CC models are as in Fig. 2, with constant (green) and variable parameters in the carbon cycle (red). Results from the C4MIP models (Friedlingstein et al. 2006; dots) and their mean (circle) are shown for the uncoupled (blue) and coupled carbon cycle (red). The 5\%-95\% ranges from PDFs derived with the Bern EMIC (Knutti et al. 2003) without (uncoupled, blue) and with (coupled, red) carbon cycle feedbacks are shown for the case where climate sensitivity is only constrained by observations (as in Fig. 2).

ferences in forcings are not significant when averaged across all models. The differences in projections from the TAR are attributable to different forcing assumptions in the MAGICC model versus the CMIP3 AOGCMs, and do not reflect a change in our understanding of the sum of the relevant physical feedback processes that affect the transient warming.

\section{d. The effect of uncertainties in the carbon cycle feedbacks}

The overall range of different methods is larger than the CMIP3 AOGCM range, because (as noted above) the AOGCM simulations do not span the full possible uncertainty range and do not sample carbon cycle uncertainties. Climate impacts on the carbon cycle are important and very likely provide a positive feedback on temperature, with warming leading to a reduced fraction of $\mathrm{CO}_{2}$ emissions being taken up by the ocean and terrestrial biosphere, and an increased fraction of the emissions staying airborne, thus causing more warming than would be produced if the carbon cycle were insensitive to climate change (Joos et al. 2001; Plattner et al. 2001; Friedlingstein et al. 2006). Figure 4 
shows uncertainty ranges from different studies and models with no carbon cycle feedbacks (i.e., constant climate prescribed for the carbon cycle), with feedbacks but fixed parameters in the carbon cycle, and with feedbacks and their uncertainty considered by perturbing parameters in the carbon cycle in different simulations. Results are shown from single models with perturbed parameters in the carbon cycle (Bern2.5CC and MAGICC), from a probabilistic study accounting for carbon cycle feedbacks with a simple feedback term (Knutti et al. 2003) and for a set of structurally different C4MIP coupled carbon cycle models (Friedlingstein et al. 2006). Independent of model and carbon cycle complexity included, these results indicate that uncertainties arising from climate feedbacks on the carbon cycle are less important than uncertainties associated with future emissions and climate sensitivity (see Fig. 2), and have little effect on the lower bound of the projected range, but affect the upper bound more strongly, consistent with earlier studies (Wigley and Raper 2001; Knutti et al. 2003).

\section{Conclusions}

On average the latest generation of CMIP3 AOGCMs used in AR4 (Meehl et al. 2007) projects changes of global temperature similar to the AOGCMs used in the TAR (Cubasch et al. 2001). Best-guess projections in degrees Celsius and likely ranges (i.e., $>66 \%$ probability, in brackets) given in AR4 (Meehl et al. 2007) for the six illustrative SRES scenarios are B1: 1.8 (1.1-2.9), B2: 2.4 (1.4-3.8), A1T: 2.4 (1.4-3.8), A1B: 2.8 (1.7-4.4), A2: 3.4 (2.0-5.4), and A1FI: 4.0 (2.4-6.4), while the TAR combined all six scenarios into a single projected range of $1.4^{\circ}-5.8^{\circ} \mathrm{C}$. For the six scenarios considered here, the relative contributions of scenario uncertainty and climate response uncertainty are similar. However, because of the very different nature of uncertainties in emissions scenarios versus uncertainties in the model response, the lack of probabilities assigned to scenarios, and because only a limited set of nonintervention scenarios is considered here, aggregating temperature ranges across scenarios should be avoided. Differences of a few tenths of a degree between the best-guess values given in AR4 versus those in the TAR for the year 2100 are mainly due to different forcing assumptions in the MAGICC model used in the TAR compared to the CMIP3 AOGCMs.

The combined information given in Fig. 2 from many different quantitative methods with a variety of models and statistical methods, considering uncertainties in radiative forcing, climate feedbacks, ocean heat uptake, and the carbon cycle, suggest an uncertainty in pro- jections larger than that covered by the AOGCM ensemble of opportunity, and larger than the range indicated in the IPCC TAR. The reason for the larger uncertainty is that uncertainties in the carbon cycleclimate feedbacks as well as structural uncertainties in both climate models and probabilistic methods are considered here when aggregating all results, while those were not considered in the TAR. We argue that the concept of a constant relative uncertainty across scenarios (irrespective of what percentage is chosen) is well supported by different studies, and provides a simple way of giving policy relevant information. The concept of constant relative uncertainty is also very likely to hold for other nonmitigation scenarios that have similar characteristics as the six illustrative SRES scenarios considered here (i.e., other scenarios approximately within the SRES range), thus providing an easy way of estimating uncertainties in global temperature projections without running many model simulations. Uncertainties tend to be slightly asymmetric in many studies, with the lower bound of the expected temperature increase being comparably well constrained and the upper bound being more uncertain. The larger uncertainty on the upper bound is caused by uncertainties in carbon cycle-climate feedbacks (Plattner et al. 2008), which are most relevant for high warming, and by the more uncertain upper bound on climate sensitivity. For the same reasons, the agreement of different studies is better for the lower than for the upper bound.

This set of projections reviewed here is based on the current understanding of climate feedbacks and uncertainties in the carbon cycle-climate interaction. While uncertainties in the former are relatively well quantified on the time scales considered here, parts of the latter are still poorly understood and not generally included in carbon cycle models (Friedlingstein et al. 2006; Plattner et al. 2008). In the terrestrial biosphere, for example, there is a likely temperature feedback on the methane gas cycle and the possibility that melting permafrost might cause the release of greenhouse gases into the atmosphere. Also, the effect of $\mathrm{CO}_{2}$ fertilization on plants might be smaller than previously thought (e.g., Körner et al. 2005). For the ocean carbon sink, changes in the atmospheric circulation may have an effect on the carbon uptake of the Southern Ocean (Le Quéré et al. 2007), an example of an effect that is unlikely to be captured in relatively simple carbon cycle models. Gruber et al. (2004) provide a detailed review of potential surprises in the biogeochemical cycles. These are examples of structural uncertainties, where some processes might be entirely missing in the biogeochemistry models. It is important to note that most of those poorly understood feedbacks are likely to be 
positive rather than negative, and therefore have the potential to raise the upper bound of our temperature uncertainties. The uncertainty in those processes is particularly large for high emission scenarios and associated large climate change, where there is no analogous situation in the past and present where models can be evaluated.

The results reviewed here also show that the contribution of structural uncertainty to temperature projections is quite large. There is no statistical method so far to formally combine the different lines of evidence. This makes the interpretation of probabilistic projections not as straightforward as a PDF from a single method might suggest.

Acknowledgments. We thank R. Furrer, G. Harris, and M. Collins for providing data. This study was supported in part by the Office of Biological and Environmental Research, U.S. Department of Energy, as part of its Climate Change Prediction Program, and in part by the Weather and Climate Impact Assessment Initiative at the National Center for Atmospheric Research. RK was supported by the Swiss National Science Foundation; MM by the German Academic Exchange Service DAAD; PAS by the U. K. Department for Environment, Food and Rural Affairs under Contract PECD 7/12/37; and TFS and GKP by the Swiss National Science Foundation (NCCR Climate) and the Swiss Federal Office for the Environment. Members of International ad hoc Detection and Attribution Group acknowledge support from NOAA Climate Program Office and the DOE Office of Science, Office of Biological and Environmental Research. We acknowledge the modeling groups for making their simulations available for analysis, the Program for Climate Model Diagnosis and Intercomparison (PCMDI) for collecting and archiving the CMIP3 model output, and the WCRP Working Group on Coupled Modelling (WGCM) for organizing the model data analysis activity. The WCRP CMIP3 multimodel dataset is supported by the Office of Science, U.S. Department of Energy.

\section{REFERENCES}

Allen, M. R., and Coauthors, 2006: Observational constraints on climate sensitivity. Avoiding Dangerous Climate Change, $\mathrm{H}$. J. Schellnhuber et al., Eds., Cambridge University Press, 281289.

Andronova, N. G., and M. E. Schlesinger, 2001: Objective estimation of the probability density function for climate sensitivity. J. Geophys. Res., 106, 22 605-22 612.

Annan, J. D., and J. C. Hargreaves, 2006: Using multiple observationally-based constraints to estimate climate sensitivity. Geophys. Res. Lett., 33, L06704, doi:10.1029/2005GL025259.

Collins, W. D., and Coauthors, 2006: Radiative forcing by well- mixed greenhouse gases: Estimates from climate models in the Intergovernmental Panel on Climate Change (IPCC) Fourth Assessment Report (AR4). J. Geophys. Res., 111, D14317, doi:10.1029/2005JD006713.

Cubasch, U., and Coauthors, 2001: Projections of future climate change. Climate Change 2001: The Scientific Basis, J. T. Houghton et al., Eds., Cambridge University Press, 525-582.

Forest, C. E., P. H. Stone, A. P. Sokolov, M. R. Allen, and M. D. Webster, 2002: Quantifying uncertainties in climate system properties with the use of recent climate observations. Science, 295, 113-117.

Forster, P. M. D., and K. E. Taylor, 2006: Climate forcings and climate sensitivities diagnosed from coupled climate model integrations. J. Climate, 19, 6181-6194.

Frame, D. J., B. B. B. Booth, J. A. Kettleborough, D. A. Stainforth, J. M. Gregory, M. Collins, and M. R. Allen, 2005: Constraining climate forecasts: The role of prior assumptions. Geophys. Res. Lett., 32, L09702, doi:10.1029/2004GL022241.

, D. A. Stone, P. A. Stott, and M. R. Allen, 2006: Alternatives to stabilization scenarios. Geophys. Res. Lett., 33, L14707, doi:10.1029/2006GL025801.

Friedlingstein, P., and Coauthors, 2006: Climate-carbon cycle feedback analysis: Results from the $\mathrm{C}^{4} \mathrm{MIP}$ model intercomparison. J. Climate, 19, 3337-3353.

Furrer, R., S. R. Sain, D. Nychka, and G. A. Meehl, 2007a: Multivariate Bayesian analysis of atmosphere-ocean general circulation models. Environ. Ecol. Stat., 14, 249-266.

- R. Knutti, S. R. Sain, D. W. Nychka, and G. A. Meehl, 2007b: Spatial patterns of probabilistic temperature change projections from a multivariate Bayesian analysis. Geophys. Res. Lett., 34, L06711, doi:10.1029/2006GL027754.

Gruber, N., and Coauthors, 2004: The vulnerability of the carbon cycle in the 21st century: An assessment of carbon-climatehuman interactions. The Global Carbon Cycle: Integrating Humans, Climate, and the Natural World, C. B. Field and M. R. Raupach, Eds., Island Press, 45-76.

Hall, J., G. Fu, and J. Lawry, 2007: Imprecise probabilities of climate change: Aggregation of fuzzy scenarios and model uncertainties. Climatic Change, 81, 265-281.

Hansen, J., A. Lacis, D. Rind, G. L. Russell, P. Stone, I. Fung, R. Ruedy, and J. Lerner, 1984: Climate sensitivity: Analysis of feedback mechanisms. Climate Processes and Climate Sensitivity, J. Hansen and T. Takahashi, Eds., Amer. Geophys. Union, 130-163.

, I. Fung, A. Lacis, D. Rind, S. Lebedeff, R. Ruedy, G. Russell, and P. Stone, 1988: Global climate changes as forecast by Goddard Institute for Space Studies 3-dimensional model. J. Geophys. Res., 93, 9341-9364.

— phys. Res., 110, D18104, doi:10.1029/2005JD005776.

Harris, G., D. M. H. Sexton, B. B. B. Booth, M. Collins, J. M. Murphy, and M. J. Webb, 2006: Frequency distributions of transient regional climate change from perturbed physics ensembles of general circulation model simulations. Climate Dyn., 27, 357-375.

Hegerl, G. C., T. J. Crowley, W. T. Hyde, and D. J. Frame, 2006: Climate sensitivity constrained by temperature reconstructions over the past seven centuries. Nature, 440, 1029-1032.

Houghton, J. T., Y. Ding, D. J. Griggs, M. Noguer, P. J. van der Linden, X. Dai, K. Maskell, and C. A. Johnson, Eds., 2001: Climate Change 2001: The Scientific Basis. Cambridge University Press, $881 \mathrm{pp}$.

Jones, P. D., and A. Moberg, 2003: Hemispheric and large-scale 
surface air temperature variations: An extensive revision and an update to 2001. J. Climate, 16, 206-223.

Joos, F., G. K. Plattner, T. F. Stocker, O. Marchal, and A. Schmittner, 1999: Global warming and marine carbon cycle feedbacks and future atmospheric $\mathrm{CO}_{2}$. Science, 284, 464467.

, I. C. Prentice, S. Sitch, R. Meyer, G. Hooss, G.-K. Plattner, S. Gerber, and K. Hasselmann, 2001: Global warming feedbacks on terrestrial carbon uptake under the Intergovernmental Panel on Climate Change (IPCC) emission scenarios. Global Biogeochem. Cycles, 15, 891-908.

Knutti, R., T. F. Stocker, and D. G. Wright, 2000: The effects of subgrid-scale parameterizations in a zonally averaged ocean model. J. Phys. Oceanogr., 30, 2738-2752.

,-- , F. Joos, and G.-K. Plattner, 2002: Constraints on radiative forcing and future climate change from observations and climate model ensembles. Nature, 416, 719-723.

,,,--- and -2003 : Probabilistic climate change projections using neural networks. Climate Dyn., 21, 257-272.

—, F. Joos, S. A. Müller, G.-K. Plattner, and T. F. Stocker, 2005: Probabilistic climate change projections for $\mathrm{CO}_{2}$ stabilization profiles. Geophys. Res. Lett., 32, L20707, doi:10.1029/ 2005 GL023294.

Körner, C., and Coauthors, 2005: Carbon flux and growth in mature deciduous forest trees exposed to elevated $\mathrm{CO}_{2}$. Science, 309, 1360-1362.

Kriegler, E., and H. Held, 2005: Utilizing belief functions for the estimation of future climate change. Int. J. Approx. Reason, 39, 185-209.

Le Quéré, C., and Coauthors, 2007: Saturation of the Southern Ocean $\mathrm{CO}_{2}$ sink due to recent climate change. Science, 316, 1735-1738.

Levitus, S., J. I. Antonov, T. P. Boyer, and C. Stephens, 2000: Warming of the world ocean. Science, 287, 2225-2229.

Meehl, G. A., W. M. Washington, W. D. Collins, J. M. Arblaster, A. Hu, L. E. Buja, W. G. Strand, and H. Teng, 2005: How much more global warming and sea level rise? Science, 307, 1769-1772.

- and Coauthors, 2007: Global climate projections. Climate Change 2007: The Physical Science Basis, S. Solomon et al., Eds., Cambridge University Press, 747-845.
Nakicenovic, N., and R. Swart, 2000: Special Report on Emissions Scenarios. Cambridge University Press, $570 \mathrm{pp}$.

Plattner, G.-K., F. Joos, T. F. Stocker, and O. Marchal, 2001: Feedback mechanisms and sensitivities of ocean carbon uptake under global warming. Tellus, 53B, 564-592.

—_, and Coauthors, 2008: Long-term climate commitments projected with climate-carbon cycle models. J. Climate, in press.

Schlesinger, M., 1986: Equilibrium and transient climatic warming induced by increased atmospheric $\mathrm{CO}_{2}$. Climate Dyn., 1, 3551.

Siegenthaler, U., and H. Oeschger, 1984: Transient temperature changes due to increasing $\mathrm{CO}_{2}$ using simple models. Ann. Glaciol., 5, 153-159.

Stott, P. A., and J. A. Kettleborough, 2002: Origins and estimates of uncertainty in predictions of twenty-first century temperature rise. Nature, 416, 723-726.

—, J. F. B. Mitchell, M. R. Allen, T. L. Delworth, J. M. Gregory, G. A. Meehl, and B. D. Santer, 2006: Observational constraints on past attributable warming and predictions of future global warming. J. Climate, 19, 3055-3069.

Tebaldi, C., and R. Knutti, 2007: The use of the multi-model ensemble in probabilistic climate projections. Philos. Trans. Roy. Soc. London, 365A, 2053-2075.

Tomassini, L., P. Reichert, R. Knutti, T. F. Stocker, and M. E. Borsuk, 2007: Robust Bayesian uncertainty analysis of climate system properties using Markov chain Monte Carlo methods. J. Climate, 20, 1239-1254.

Wigley, T. M. L., 2004: Modeling climate change under no-policy and policy emissions pathways. The Benefits of Climate Change Policies: Analytical and Framework Issues, J. Corfee Morlot and S. Agrawala, Eds., OECD Publications, 221-248. - 2005: The climate change commitment. Science, 307, 17661769.

- , and M. E. Schlesinger, 1985: Analytical solution for the effect of increasing $\mathrm{CO}_{2}$ on global mean temperature. Nature, 315, 649-652.

— , and S. C. B. Raper, 2001: Interpretation of high projections for global-mean warming. Science, 293, 451-454.

— , R. Richels, and J. A. Edmonds, 1996: Economic and environmental choices in the stabilization of atmospheric $\mathrm{CO}_{2}$ concentrations. Nature, 379, 240-243. 